\title{
A ROBUST CAPACITIVE PRESSURE SENSOR ARRAY
}

\author{
Jeong-Bong Lee, Sung-Pil Chang, and Mark G. Allen \\ School of Electrical and Computer Engineering, Georgia Institute of Technology, Atlanta, Georgia 30332-0269 \\ Phone: (404) 894-8807, Fax: (404) 894-5028, e-mail: jeong-bong.lee@ece.gatech.edu
}

\begin{abstract}
In this work, robust substrates, such as a stainless steel, are studied as substrates for micromachined devices. The use of robust substrates may allow for the co-fabrication of micromachined sensors and sensor packages. Lamination combined with traditional micromachining processes has been investigated as a suitable technology for these investigations. To illustrate this technology, a capacitive pressure sensor array has been designed, fabricated, and characterized using a stainless steel substrate, Kapton film as a diaphragm, and an electroplated nickel fixed back electrode. The net capacitance change over the applied pressure range ( 0 to $34 \mathrm{kPa}$ ) was approximately $0.23 \mathrm{pF}$.
\end{abstract}

\section{INTRODUCTION}

Micromachined devices have been primarily realized using silicon substrates [1]. In this work, the use of robust substrates (such as stainless steel) as suitable starting points for both bulk and surface micromachined structures, as well as the possibility of the substrate forming essential structural components of the device package, have been investigated. In addition to new robust substrates, alternative fabrication techniques such as lamination have been studied. To illustrate these principles, a robust capacitive pressure sensor array has been fabricated with ultimate application to flow control problems, such as the guidance of unmanned airborne vehicles (UAV). An important attribute of this design is that only the steel plate and the pressure sensor inlet is exposed to the flow; i.e., the sensor is self-packaged.

\section{THOERY AND DESIGN}

The sensor device concept is based on the pressure-induced deflection of a metallized diaphragm and the subsequent measurement of the capacitance between this deflecting diaphragm and a fixed surface micromachined backplate suspended over the diaphragm.

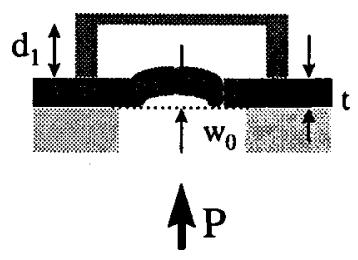

Figure 1. A schematic diagram of the side-view of the capacitive pressure sensor.

Figure 1 shows a schematic diagram of the side-view of the device, where $d_{1}$ is an initial gap distance between the fixed back electrode and the flexible diaphragm electrode, $w_{0}$ is the deflection at the center of the diaphragm, $t$ is the thickness of the flexible Kapton diaphragm, and $P$ is the applied pressure. For analytical modeling, several assumptions have been made: (a) neglect stretching on the diaphragm, (b) neglect thickness of metallic electrode on the diaphragm, and (c) neglect fringing effects. The sensor capacitance can be described as follows:

$$
C_{\text {sensor }}=\varepsilon_{0} \int_{0}^{2 \pi a} \int_{0} \frac{r d r d \theta}{\left(d_{1}-w(r)\right)},
$$

where $a$ is the radius of the diaphragm, and $0 \leq r \leq a$. The solution of equation (1) can be expressed as a Taylor series expansion as follows:

$$
C_{\text {sensor }}=C_{0}\left(1+K_{1} P+K_{2} P^{2}+\cdots\right),
$$

where $C_{0}$ is the capacitance when the diaphragm is undeflected, and $K_{n}$ are constants.

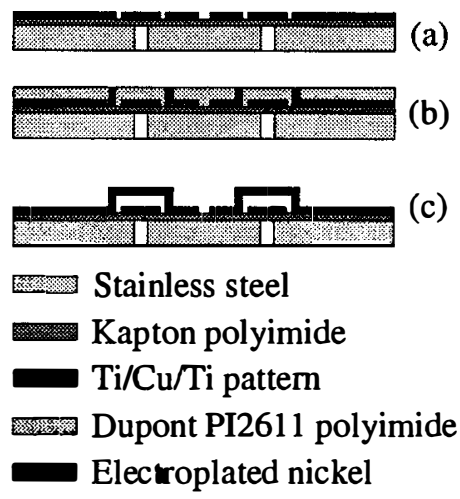

Figure 2. Fabrication sequence: (a) lamination of milled stainless steel and Kapton polyimide film and bottom electrode patterning; (b) polyimide deposition and electroplating of nickel posts; (c) backplate electroplating and removal of polyimide sacrificial layer.

\section{FABRICATION AND MEASUREMENT}

The fabrication sequence of the robust capacitive pressure sensor array is shown in Figure 2. The process began with milling an $8 * 8$ array of pressure inlet holes ( $2 \mathrm{~mm}$ diameter) in a $0.5 \mathrm{~mm}$ thick, 2 1/4 inch on a side stainless steel shim stock. Lamination of Dupont Kapton HN200 (50 $\mu \mathrm{m}$ thick) polyimide film on the stainless steel was performed using a hot press. The regions of the Kapton polyimide film over the pressure inlet holes will form the pressure-sensitive diaphragms. A Ti/Cu/Ti $(200 / 2,000 / 500 \AA$ respectively) metallic layer was deposited on the Kapton surface and patterned using a lift-off technique to form bottom electrodes, bonding pads, and electroplating seed layers for backplate posts (see Figure 3). Multiple layers of Dupont polyimide PI2611 were coated on the patterned metallic layer, hard cured (resulting in a final thickness of $36 \mu \mathrm{m}$ ), and anisotropically dry etched using reactive ion etching to form plating molds. Nickel was electroplated through the polyimide molds to form backplate posts. Another $\mathrm{Ti} / \mathrm{Cu} / \mathrm{Ti}$ metallic seed layer was deposited and thick photoresist (Shipley PR 5740) was patterned as electroplating 
molds for the fixed backplates. Electroplated nickel backplates approximately $15 \mu \mathrm{m}$ thick were formed and the photoresist molds and metallic seed layers were removed. Finally, an isotropic dry etch process was performed using a barrel plasma etcher (a gas mixture of $\mathrm{O}_{2}$ and $\mathrm{CF}_{4}$ ) to remove the polyimide molds for backplate posts as well as polyimide sacrificial layers to create air cavities between the fixed backplates and the pressure sensitive Kapton polyimide diaphragms. Figure 4 shows photographs of a fabricated pressure sensor array, where (a) gives a side-view and (b) gives a close-up view of the gap defined between the backplate and the diaphragm.

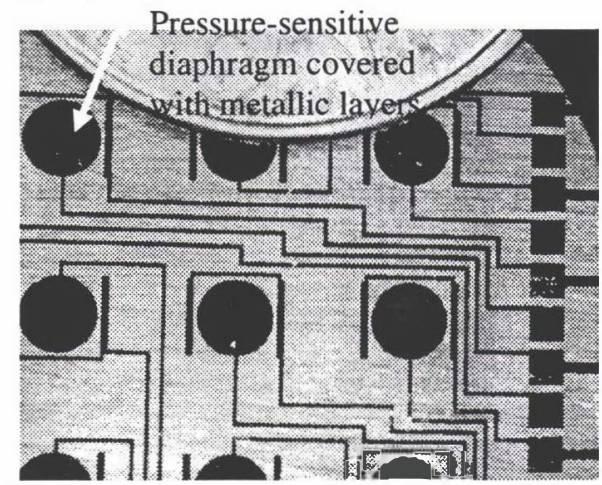

Figure 3. A photograph of top-view of the metallic seed layer which corresponds to the fabrication sequence shown in Figure 2 (a). The pressure-sensitive diaphragms are located underneath the circular metallic patterns. The array is shown next to a penny for size comparison.

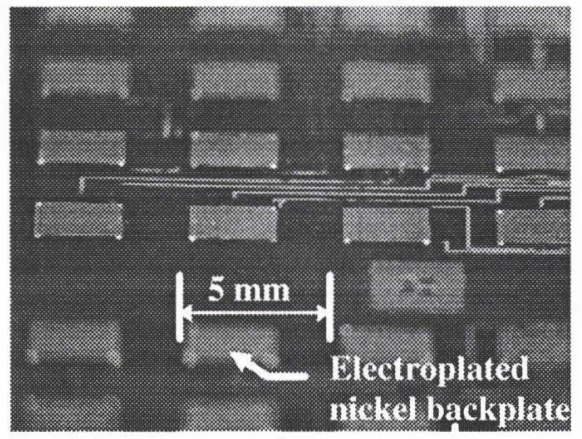

(a)

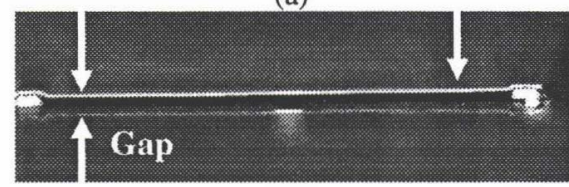

(b)

Figure 4. Photographs of fabricated pressure sensor array: (a) A side-view of the pressure sensor array; (b) a close-up view of the gap (approximately $36 \mu \mathrm{m}$ ) defined between electroplated nickel backplate and pressure sensitive Kapton diaphragm.

The capacitance of individual sensors was measured using a Keithley 3322 LCZ meter. Measured capacitances for undeflected pressure sensors were in the range from $11.02 \mathrm{pF}$ to $17.52 \mathrm{pF}$ depending on the length of interconnections between bonding pads and sensors. It was observed that capacitance monotonically increases for increasing applied pressure, meaning that the pressure sensitive Kapton diaphragm deflects toward the fixed backplates. Over an applied pressure range from 0 to $34 \mathrm{kPa}$, the net capacitance change was approximately $0.23 \mathrm{pF}$. Theoretical and measured net capacitance change over the applied pressure range are compared in Figure 5. Theoretical data shown in Figure 5 is based on initial gap of $33 \mu \mathrm{m}$. There is approximately $10 \%$ difference between the measured gap and the theoretical gap value, and this difference could be explained by fringing effect of the sensor capacitance. The measured values of relative capacitance change are in the range from $1.54 \%$ to $2.14 \%$. The values of capacitance changed produced by the sensors are easily measurable in their current form; however, if required, the parasitic effect can be reduced using an unchanging reference capacitance and appropriate subtraction circuitry. By doing so, the relative capacitance change over the applied pressure range could be increased to $22.3 \%$.

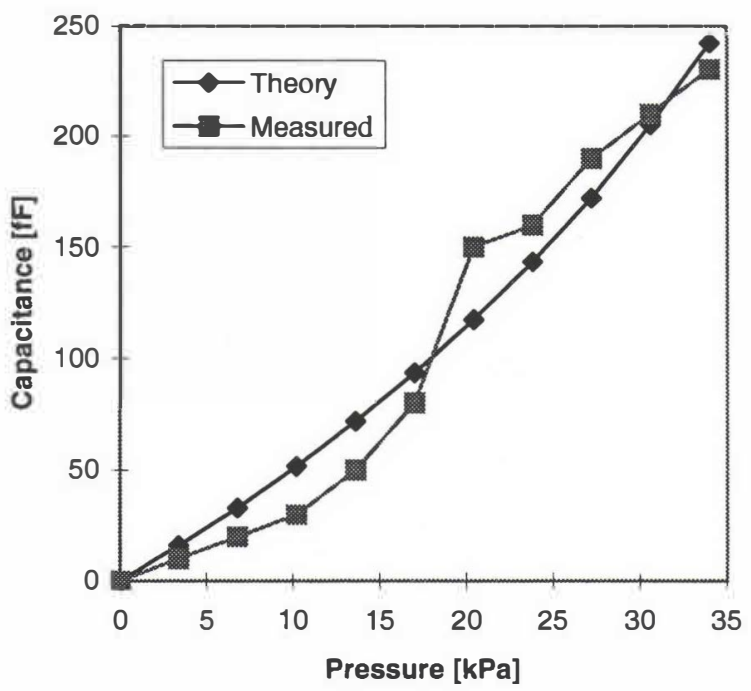

Figure 5. A comparison of theoretical and measured values of net capacitance change over the full range of applied pressure ranging from 0 to $34 \mathrm{kPa}(10 \mathrm{in}-\mathrm{Hg})$.

\section{CONCLUSIONS}

Robust materials have been studied as suitable substrates for micromachined devices. Lamination using a hot press, combined with traditional micromachining processes, has been investigated as a suitable fabrication process for the robust substrates. A capacitive pressure sensor array using a robust substrate $(500 \mu \mathrm{m}$ thick stainless steel shim stock), Kapton HN200 diaphragm, and lamination processing has been designed, fabricated, and characterized. Over the applied pressure range from 0 to $34 \mathrm{kPa}$, the net capacitance change of the pressure sensor is approximately $0.23 \mathrm{pF}$.

\section{ACKNOWLEDGMENT}

The support of the staff of the Microelectronics Research Center at Georgia Tech is acknowledged. This work was supported by Defense Advanced Research Project Agency (DARPA) and Air Force Office of Scientific Research (AFOSR) under Contract F49620-97-1-0519.

\section{REFERENCES}

1. H. Baltes, "CMOS Micro Electro Mechanical Systems," Sensors and Materials, v. 9, n. 6, p.331-346 (1997). 